\title{
Características morfométricas e dendrocronológicas de Zanthoxylum rhoifolium Lam em fragmento de Floresta Ombrófila Mista Aluvial
}

\author{
Morphometric and dendrochronological characteristics of Zanthoxylum \\ rhoifolium Lam in Mixed Alluvial Ombrophylous Forest fragment
}

\author{
Ana Paula Vantroba ${ }^{1}$ (D), Iris Cristina Bertolini ${ }^{1}$ (D), Talyta Mytsuy Zanardini Galeski Sens ${ }^{1}$ (D), \\ Luciano Farinha Watzlawick ${ }^{1}$ (1D, Jaqueline Aparecida Schran ${ }^{1}$ (ib, Beatriz Cristina Pedroso ${ }^{1}$ (i) \\ ${ }^{1}$ Universidade Estadual do Centro-Oeste - UNICENTRO, Guarapuava, PR, Brasil
}

Como citar: Vantroba, A. P., Bertolini, I. C., Sens, T. M. Z. G., Watzlawick, L. F., Schran, J. A., \& Pedroso, B. C. (2020). Características morfométricas e dendrocronológicas de Zanthoxylum rhoifolium Lam em fragmento de Floresta Ombrófila Mista Aluvial. Scientia Forestalis, 48(127), e3338. https://doi.org/10.18671/scifor.v48n127.10

\begin{abstract}
Resumo
Zanthoxylum rhoifolium Lam é uma espécie de ampla ocorrência nas florestas brasileiras e apresenta potencial para múltiplos usos de sua madeira. Contudo, para o maior conhecimento desta espécie, são necessários estudos de sua autoecologia e dinâmica na floresta. Com intuito de contribuir com os avanços dos estudos de espécies florestais nativas, buscou-se avaliar as características morfométricas para as três posições sociológicas: dominada, intermediária e dominante, e também suas características dendrocronológicas em um fragmento de Floresta Ombrófila Mista Aluvial, situado na área urbana do município de Guarapuava, PR. Para avaliação da morfometria foi medido o diâmetro a altura do peito, altura total e de inserção da copa, e os oito raios de projeção de copa. Os dados de incremento foram obtidos pela análise de 74 baguetas, coletadas com a utilização do trado de Pressler e correlacionados com as variáveis climáticas. Verificou-se correlação significativa a nível de $1 \%$ de probabilidade do crescimento com a precipitação. Os índices de esbeltez, saliência e abrangência tiveram grande variabilidade, com valores de 35,71 a 166,32, 13,04 a 47,25 e 0,13 a 0,96 respectivamente. A espécie Z. rhoifolium apresentou alta correlação do DAP com o grau de esbeltez, e da altura total com o comprimento da copa.
\end{abstract}

Palavras-chave: Floresta de Araucária; Crescimento; Anéis de crescimento; Variáveis climáticas.

\begin{abstract}
Zanthoxylum rhoifolium Lam is a species of wide occurrence in Brazilian forests and its wood has potential multiple uses. However, for the better knowledge of this species, studies of its ecology and dynamics in the forest are necessary. In order to contribute to the advances of native forest species studies, we sought to evaluate the morpho-metric characteristics of the tree's three sociological positions: dominated, intermediate and dominant, as well as its dendro-chronological characteristics in a fragment of Alluvial Mixed Ombrophilous Forest, located in the urban area of Guarapuava, PR. To evaluate the morphometry, the diameter at breast height, total and canopy insertion height, and the eight canopy projection radii were measured. The increment data were obtained by the analysis of 74 increment cores, collected using the Pressler auger and correlated with the climatic variables. Significant correlation was found at $1 \%$ probability of growth with precipitation. The leanness, prominence and coverage indices had great variability, with values from 35.71 to $166.32,13.04$ to 47.25 and 0.13 to 0.96 respectively. The species $Z$. rhoifolium showed a high correlation between $\mathrm{DBH}$ and the degree of slenderness, and the total height with the crown length.
\end{abstract}

Keywords: Araucaria forest; Growth; Growth rings; Climatic variables.

Fonte de financiamento: CAPES - Coordenação de Aperfeiçoamento de Pessoal de Nível Superior.

Conflito de interesse: Nada a declarar.

Autor correspondente: paulavantroba@hotmail.com

Recebido: 15 abril 2019.

Aceito: 2 setembro 2019

Editor: Paulo Henrique Müller Silva.

(c) Este é um artigo publicado em acesso aberto (Open Access) sob a licença Creative Commons Attribution, que permite uso, distribuição e reprodução em qualquer meio, sem restrições desde que o trabalho original seja corretamente citado. 


\section{INTRODUÇÃO}

A espécie Zanthoxylum rhoifolium ocorre de forma natural em todos os estados brasileiros, tendo condições ideais para se desenvolver na Floresta Ombrófila Mista, em regiões de clima frio (Carvalho, 2006). Considerando que existem diversos estudos a fim de compreender a dinâmica como um todo, ainda não se tem conhecimento a fundo do potencial e ritmo de crescimento de cada uma das espécies em seu ambiente natural.

O crescimento das árvores é influenciado por diversos fatores ambientais entre eles temperatura, precipitação, insolação, características físicas, químicas e biológicas do solo, inclinação, altitude e exposição do terreno, e também com fatores biológicos, como a competição.

Um dos estudos ecológicos importantes para o conhecimento do crescimento das árvores são às relações morfométricas, as quais permitem perceber a sua resposta ao ambiente em termos de ocupação de espaço, de competição e, consequentemente de dinâmica dentro de uma floresta, além de fornecer subsídio para determinar o manejo adequado de acordo com o local em que a espécie está inserida visando o baixo impacto na floresta natural, promovendo assim, a sua conservação e expansão da espécie (Orellana \& Koehler, 2008).

A dendrocronologia também pode ser utilizada para acompanhar o crescimento das árvores, pois estuda as camadas de crescimento anuais das árvores, que são chamadas de anéis de crescimento que são um dos principais registros biológicos das condições climáticas e ambientais do passado (Santos et al., 2013). Os anéis de crescimento estão ligados ao crescimento secundário das plantas arbóreas que podem ser divididos em duas partes: lenho primaveril (inicial) e lenho outonal (estival).

Contudo, o objetivo desse trabalho foi analisar as características morfométricas e a dendrocronologia de Zanthoxylum rhoifolium em fragmento de Floresta Ombrófila Mista Aluvial (FOMA) e correlacioná-las com as variáveis climáticas locais (FOMA).

\section{MATERIAL E MÉTODOS}

\section{Caracterização da área}

A área de estudo encontra-se no município de Guarapuava que está localizada na parte sul do Terceiro Planalto do Paraná, sendo esta região delimitada pelos rios Iguaçu e Piquiri, denominada Bloco do Planalto de Guarapuava, com altitude de aproximadamente 1020 metros em relação ao nível do mar.

A área de estudo compreende um fragmento de 11,5 ha de Floresta Ombrófila Mista Aluvial (FOMA), conforme classificação do Instituto Brasileiro de Geografia e Estatística (2012). O clima na região de Guarapuava é do tipo Subtropical Úmido Mesotérmico- Cfb, conforme a classificação de Köppen, sem estação seca definida. O mês de julho é o mais seco e com a menor temperatura mínima, como mostra a Figura 1.

Terceiro planalto faz parte da formação geológica da bacia do Rio Paraná, denominada de Serra Geral ou derrame de Trapp. Dentre os tipos de solo destacam-se: Latossolo Bruno, Nitossolo Bruno, Hidromórficos, Cambissolos e Neossolos Litólicos (Empresa Brasileira de Pesquisa Agropecuária, 2013).

\section{Coleta, preparação e análise dos rolos de incremento}

Foram coletadas amostras não destrutivas em 37 indivíduos de Zanthoxylum rhoifolium no DAP $(1,30 \mathrm{~m})$, com o auxílio do trado de Pressler de $5 \mathrm{~mm}$ extraindo-se dois rolos de incremento por árvore. Após a coleta, os rolos foram fixados com cola branca em "porta-rolos" de madeira para promover a secagem em temperatura ambiente por um período de quatro semanas. Na sequência, as amostras foram polidas com lixas para facilitar a leitura dos anéis de crescimento. 


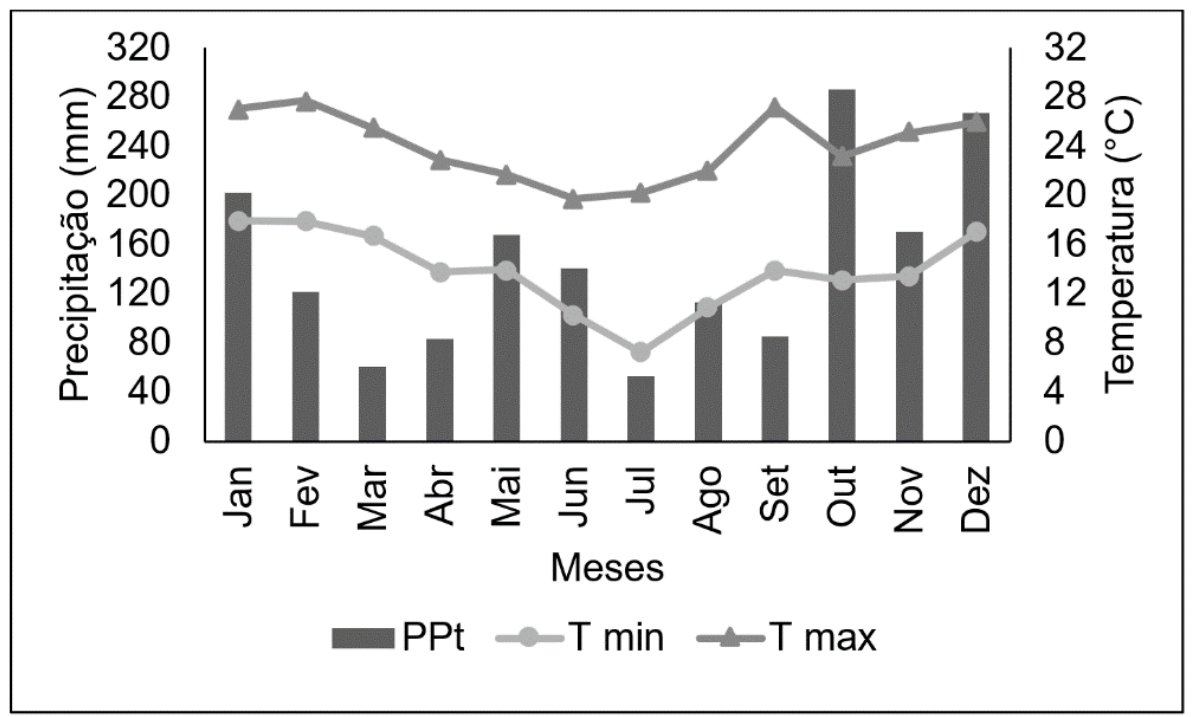

Figura 1. Climatograma da média mensal de precipitação e temperatura mínima e máxima para o município de Guarapuava, PR.

As amostras foram digitalizadas ao lado de uma régua com um scanner de mesa e as imagens obtidas foram visualizadas no software Image 2.0. A mensuração do incremento foi realizada com o auxílio da ferramenta de distância. Os dados obtidos foram exportados para software Microsoft Excel para realização da datação cruzada.

A verificação estatística das séries foi realizada com software COFECHA (Holmes, 1983), por ser exclusivo para análises dendrocronológicas, além de ajustar as séries para identificar as amostras e os segmentos que apresentam problemas de mensuração do crescimento. $O$ COFECHA gera um modelo chamado de série Master, que é usado para verificar por meio da correlação de Pearson, se há uma correlação significativa com os dados reais. As árvores que não apresentaram correlação significativa foram excluídas.

O software ARSTAN foi utilizado para a remoção das tendências de crescimento de cada amostra com o ajuste de uma função exponencial negativa e da aplicação de uma função spline cúbica. Neste software, a série Master é padronizada para eliminar as tendências de crescimento e gerar uma série denominada Standard.

Na sequência do processo de análise, os dados de crescimento médio anual foram submetidos a correlação de Pearson para verificar a relação com os índices de temperatura, precipitação e insolação por meio do software SPSS, versão 2.2 (versão teste) para verificar a relação entre o crescimento e as variáveis climáticas. Os dados climáticos foram obtidos da estação meteorológica da Universidade Estadual do Centro Oeste, distante aproximadamente 100 metros da área de estudo. O período de análise foi de 1973 a 2017. Os dados climáticos foram ajustados com o período de crescimento da planta, sendo considerado o início do crescimento em agosto do ano anterior até julho do ano subsequente.

\section{Análise morfométricas}

Foram amostrados 37 indivíduos de Zanthoxylum rhoifolium em parcelas permanentes, no mês de novembro de 2017. Foram medidos o diâmetro a altura do peito (DAP), altura total $(\mathrm{H})$, altura de inserção da copa (HIC) e os oito raios de projeção de copa nos sentidos norte, noroeste, sul, sudeste, leste, nordeste, oeste e noroeste, do tronco até o limite da copa.

Com a medida dos oito raios da copa foi calculado o diâmetro da copa (DC). As alturas das árvores foram medidas com o hipsômetro (Vertex III). Para as medições dos DAP e dos raios de copa utilizou-se fita métrica.

A posição sociológica foi determinada de acordo com a posição da árvore ocupada no estrato vertical da floresta e o respectivo grau de exposição da copa por luz, sendo classificadas em três posições sociológicas: árvores dominadas que ocupavam o estrato 
inferior com baixa exposição da copa por luz; árvores intermediárias que situavam-se no estrato intermediário com média exposição da copa por luz e árvores dominantes que ocupavam o estrato superior com alta exposição da copa por luz.

As variáveis morfométricas analisadas foram: altura total $(\mathrm{H})$, Altura de Inserção da Copa (HIC), DAP, Diâmetro de Copa (DC), Comprimento de Copa (CC= H-HIC), Proporção de Copa $\left(P C=C C / H^{*} 100\right)$, Grau de Esbeltez (GE= H/DAP), Índice de Saliência (IS= DC/DAP), Índice de Abrangência $(I A=D C / H)$ e Formal de Copa $(F C=D C / C C)$, conforme Burger (1939). Foi realizada correlação de Pearson entre o DAP e $\mathrm{H}$ com as demais variáveis e regressão linear entre o GE e DAP, e H e DAP. As análises foram feitas no software SPSS versão teste. Os gráficos e tabelas foram confeccionados no software Origin 8.5.

\section{RESULTADOS E DISCUSSÃO}

\section{Características Morfométricas}

A caracterização das posições sociológicas para os indivíduos de Zanthoxylum rhoifolium amostrados no local de estudo está apresentada na Figura 2.

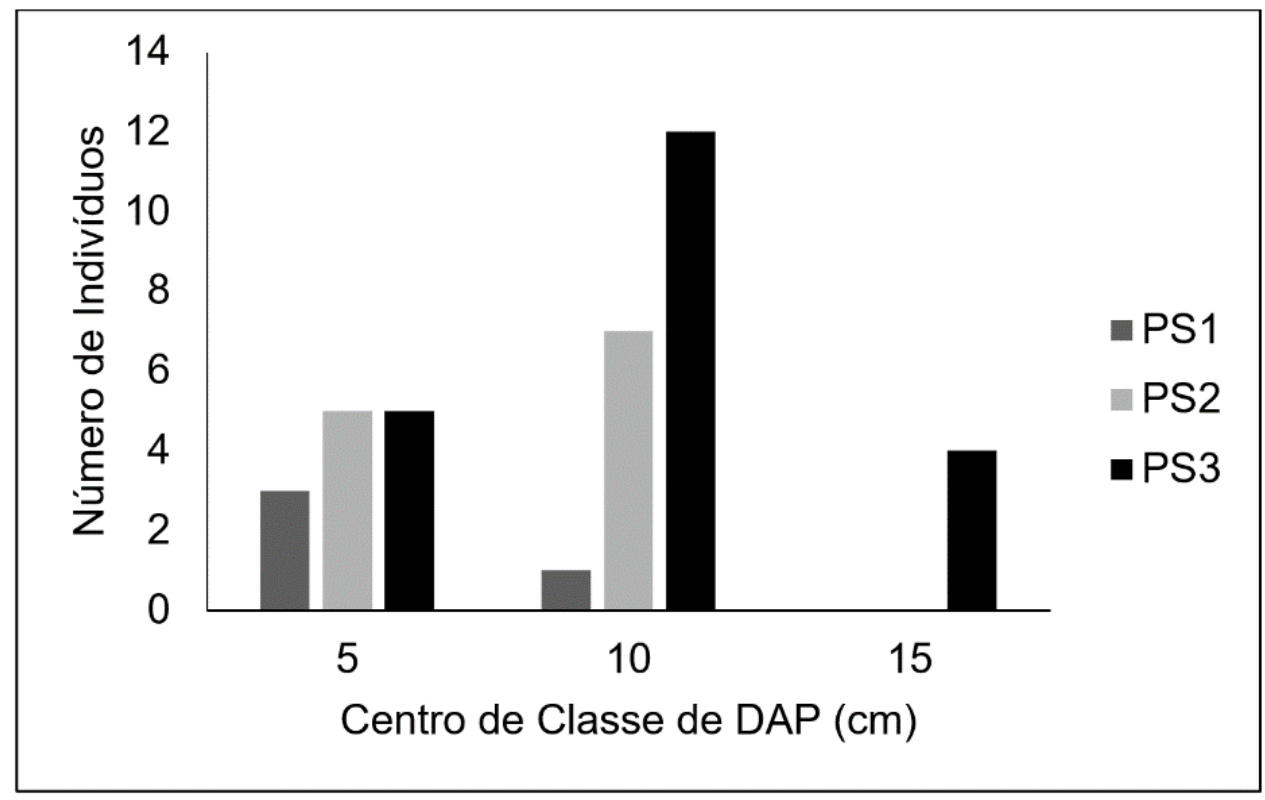

Figura 2. Distribuição diamétrica das posições sociológicas dos indivíduos de Zanthoxylum rhoifolium em um fragmento de Floresta Ombrófila Mista Aluvial em Guarapuava, PR. Onde: PS1 - Dominada; PS2Intermediária; PS3- Dominante.

Os resultados mostraram uma distribuição com maior número de indivíduos na classe de 10 a 14,9 cm. A classe de 15 a 19,9 apresentou menor quantidade de indivíduos e apenas indivíduos da posição sociológica dominante. Lamprecht (1990) afirmou que uma distribuição diamétrica regular, ou seja, quando há um número maior de indivíduos em classes menores, favorece a existência e sobrevivência das espécies regenerantes.

Resultados semelhantes foram encontrados por Costa (2011) com Araucaria angustifolia, o qual constatou que árvores com copas vigorosas e diâmetros maiores ocupam o estrato superior dentre os demais indivíduos, sendo que, a maior incidência de luz favorece seu desenvolvimento.

Os resultados das variáveis morfométricas de Zanthoxylum rhoifolium nas três posições sociológicas estão na Tabela 1. 
Tabela 1. Caracterização das variáveis morfométricas de Zanthoxylum rhoifolium nas diferentes posições sociológicas, em um fragmento de Floresta Ombrófila Mista Aluvial em Guarapuava, PR.

\begin{tabular}{|c|c|c|c|c|c|}
\hline Variáveis & PS & Média & Mínimo & Máximo & CV (\%) \\
\hline & PS1 & 8,41 & 5,76 & 10,22 & 24,29 \\
\hline \multirow[t]{3}{*}{$\mathrm{DAP}(\mathrm{m})$} & PS2 & 10,48 & 7,42 & 15,02 & 21,65 \\
\hline & PS3 & 13,90 & 5,41 & 22,41 & 36,76 \\
\hline & PS1 & 8,13 & 6,50 & 10,00 & 18,38 \\
\hline \multirow[t]{3}{*}{$H(m)$} & PS2 & 8,46 & 6,50 & 10,50 & 15,63 \\
\hline & PS3 & 8,48 & 6,50 & 11,00 & 11,42 \\
\hline & PS1 & 6,00 & 4,00 & 8,00 & 30,43 \\
\hline \multirow[t]{3}{*}{ HIC (m) } & PS2 & 4,29 & 0,50 & 8,00 & 40,19 \\
\hline & PS3 & 4,14 & 2,00 & 7,00 & 33,52 \\
\hline & PS1 & 2,49 & 2,20 & 2,80 & 12,51 \\
\hline \multirow[t]{3}{*}{$\mathrm{DC}(\mathrm{m})$} & PS2 & 3,46 & 2,10 & 4,95 & 23,63 \\
\hline & PS3 & 4,14 & 1,15 & 8,13 & 47,36 \\
\hline & PS1 & 2,13 & 1,50 & 2,50 & 22,53 \\
\hline \multirow[t]{3}{*}{ CC (m) } & PS2 & 4,17 & 2,00 & 6,00 & 30,84 \\
\hline & PS3 & 4,33 & 1,00 & 7,00 & 40,69 \\
\hline & PS1 & 27,36 & 17,65 & 38,46 & 37,00 \\
\hline \multirow[t]{3}{*}{ PC (\%) } & PS2 & 50,21 & 20,00 & 92,31 & 33,89 \\
\hline & PS3 & 50,40 & 12,50 & 76,47 & 36,08 \\
\hline & PS1 & 98,82 & 76,50 & 112,82 & 16,33 \\
\hline \multirow[t]{3}{*}{ GE } & PS2 & 82,76 & 63,03 & 112,60 & 18,61 \\
\hline & PS3 & 70,23 & 35,51 & 166,32 & 43,53 \\
\hline & PS1 & 31,45 & 22,02 & 47,25 & 34,78 \\
\hline \multirow[t]{3}{*}{ IS } & PS2 & 33,35 & 22,74 & 44,36 & 18,86 \\
\hline & PS3 & 29,46 & 13,04 & 45,10 & 27,50 \\
\hline & PS1 & 0,32 & 0,23 & 0,42 & 28,83 \\
\hline \multirow[t]{3}{*}{ IA } & PS2 & 0,41 & 0,24 & 0,58 & 23,98 \\
\hline & PS3 & 0,49 & 0,13 & 0,96 & 48,18 \\
\hline & PS1 & 1,20 & 1,09 & 1,47 & 14,86 \\
\hline \multirow[t]{2}{*}{ FC } & PS2 & 0,91 & 0,51 & 1,74 & 40,85 \\
\hline & PS3 & 1,08 & 0,29 & 2,05 & 47,42 \\
\hline
\end{tabular}

Onde: PS- Posição sociológica; PS1 - Dominada; PS2-Intermediária; PS3- Dominante; DAP- diâmetro a altura do peito; H- altura total da árvore; HIC- altura de inserção da copa; DC- diâmetro da copa; CC- comprimento da copa; PCproporção da copa; GE- grau de esbeltez; IS- índice de saliência; IA- índice de abrangência; FC- formal de copa e CVCoeficiente de Variação. Fonte: o Autor

As variáveis que apresentaram os maiores coeficientes de variação foram o DAP, Índice de Abrangência, Formal de Copa, Diâmetro de Copa, Grau de Esbeltez e Comprimento de Copa. Isso pode estar relacionado com as diferentes idades das árvores de Zanthoxylum rhoifolium. Os valores médios obtidos para o DAP foram 8,41, 10,48 e 13,90 cm para árvores das classes dominadas, intermediárias e dominantes, respectivamente.

A altura total dos indivíduos de Zanthoxylum rhoifolium na classe dominada apresentou uma média de $8,13 \mathrm{~m}$ e coeficiente de variação de 18,38\%, já as árvores dominantes apresentaram altura total média de $8,48 \mathrm{~m}$ e coeficiente de variação de 11,42\%. A altura mínima foi $6,5 \mathrm{~m}$ e a máxima $11,0 \mathrm{~m}$.

A HIC ou altura comercial do fuste apresentou valores entre 0,50 a $8 \mathrm{~m}$, sendo que os indivíduos da classe dominada apresentaram a maior HIC média, com 6 m. A variabilidade 
entre os indivíduos foi alta e o maior coeficiente de variação foi de 40,19\% para os indivíduos intermediários.

Homczinski (2017) em trabalho na Floresta Nacional de Irati-PR, obteve para a Campomanesia xanthocarpa os valores médios de $14 \mathrm{~m}$ para altura total, 7,2 m para HIC e DAP de $32,09 \mathrm{~cm}$.

Em relação ao DC dos indivíduos de Zanthoxylum rhoifolium mostrou uma variação de 1,15 a 8,13 m. Os valores médios do DC seguiram a mesma tendência do DAP, em que os indivíduos dominados apresentaram média de 2,49 m, os intermediários de 3,46 m e os dominantes de 4,14, os quais também apresentaram o maior coeficiente de variação de $(47,36 \%)$.

A PC observada em Zanthoxylum rhoifolium apresentou média de 27,36\% para as árvores dominadas e 50,4\% para as dominantes, com mínimo de 12,5\% e máximo de 92,3\%. O maior coeficiente de variação para essa variável morfométrica foi para as árvores dominadas (37\%). Essa amplitude para a PC é resultado das diferentes idades e graus de concorrência a que as árvores estão submetidas (Roman et al., 2009) e é um indicador de vitalidade, sendo que quanto maior a porcentagem, tanto mais produtiva pode ser esta árvore.

A elevada proporção de copa nas espécies, como é o caso de Zanthoxylum rhoifolium apresentam a maior parte de sua estrutura em forma de copa, tornando-se bastante dependente da radiação solar para realizar sua manutenção e desenvolvimento (Condé et al., 2013).

No que se refere ao GE, os valores observados para a espécie foram entre 35,51 e 166,32, com média de 98,82 para as árvores da classe dominada, 82,72 para as intermediárias e 70,23 para as dominantes, sendo que essa última apresentou o maior coeficiente de variação do GE (43,53\%), resultado da grande diferença entre as medidas do DAP e da altura total das árvores avaliadas. Essa variável caracteriza a instabilidade das árvores, quanto maior o GE, mais instável é a árvore, tornando-se mais sensível à quebra dos galhos e danos da copa com os ventos fortes, portanto os indivíduos dominados de Zanthoxylum rhoifolium são mais instáveis que os indivíduos dominantes.

Para Lanzarim (2016), valores superiores a 100 para GE podem ser resultados da falta de luz devido à competição entre as árvores, ocasionando a inibição do crescimento em diâmetro, as quais investem mais no crescimento em altura.

O IS observado indica que em média as copas de Zanthoxylum rhoifolium da posição sociológica dominada são 31,45 vezes maiores que o DAP e as dominantes 29,46. Os valores de IS variaram entre 13,04 e 47,25. Essa diferença do IS ocorre por ser uma relação do diâmetro de copa com o DAP, e as duas variáveis apresentaram grande variação entre as árvores avaliadas. Silveira (2014) verificou valores de IS entre 16 e 44 com média de 31 para plantas da espécie $M$. eleagnoides.

Conforme Tonini \& Arco-Verde (2005), espécies de maior crescimento em termos de volume apresentam valores entre 15 e 20 para o IS, espécies com valores superiores a 20 tendem a um baixo crescimento.

O IA apresentou valores mínimo de 0,13 e máximo de 0,96. A média para os indivíduos dominados foi de 0,32, para os intermediários 0,41 e para os dominantes 0,49, com um coeficiente de variação de 48,18\% para essa última classe. O IA tende a diminuir com a altura, porque o aumento na altura não é acompanhado, proporcionalmente, pelo aumento do DC, ou seja, o crescimento em altura ainda é maior que o crescimento do DC. Segundo Orellana \& Koehler (2008) se um povoamento for manejado pela altura das árvores, pode-se usar este índice como critério para tomada de decisão em um plano de desbaste. Silveira (2014) encontrou valor médio para o índice de abrangência para Matayba eleagnoides de 0,76.

Homczinski (2017) para a Campomanesia xanthocarpa, encontrou valores médios para DC, CC e IA de 9,5 m 7,2 m e 0,66, respectivamente.

O FC de Zanthoxylum rhoifolium apresentou valores entre 0,29 e 2,05. O valor médio para os indivíduos da classe dominada foi 1,20, para os intermediários 0,91 e para os dominantes 1,08 . 
De acordo com esses resultados encontrados para FC de Zanthoxylum rhoifolium é possível indicar que na comunidade existem dois tipos de copas, onde as árvores intermediárias possuem copas alongadas (FC é abaixo de 1) e as árvores da classe dominada e dominante apresentam copas chatas (FC acima de 1) (Durlo, 2001).

\section{Análise de correlação entre as variáveis morfométricas}

O DAP e a H são variáveis de mais fácil medição, sendo indicadas para serem usadas nesses modelos. A correlação das variáveis DAP e a $\mathrm{H}$ com as demais variáveis morfométricas foram analisadas e os resultados apresentados na Tabela 2.

Para Tonini \& Arco-Verde (2005) a medição das variáveis morfométricas consome muito tempo, ao invés disso podem ser utilizadas variáveis independentes de fácil medição, como o DAP e $\mathrm{H}$.

Tabela 2. Matriz de correlação linear de Pearson entre o diâmetro à altura do peito - DAP e a altura total - H e as variáveis morfométricas de Zanthoxylum rhoifolium.

\begin{tabular}{|c|c|c|c|c|c|c|c|c|c|c|}
\hline Variável & DAP & H & HIC & DC & CC & IA & FC & GE & IS & PC \\
\hline DAP & - & 0,26 & $\begin{array}{l}- \\
0,48^{* *}\end{array}$ & $0,80 * \star$ & $0,63 * *$ & $0,70 * *$ & 0,05 & $\begin{array}{l}- \\
0,86 * *\end{array}$ & $-0,10$ & $0,58 * *$ \\
\hline $\mathbf{H}$ & 0,26 & - & 0,31 & 0,10 & $0,37^{*}$ & $-0,22$ & $-0,26$ & 0,08 & $-0,32$ & 0,030 \\
\hline
\end{tabular}

DAP- Diâmetro a Altura do Peito; H- Altura total da árvore; HIC- Altura de Inserção da Copa; DC- Diâmetro da copa; CCComprimento da Copa; IA- Índice de Abrangência; FC- Formal da Copa; GE- Grau de Esbeltez; IS- Índice de Saliência; PCProporção da Copa. * Correlação é significativa ao nível de 0,05, ** Correlação é significativa ao nível de 0,01. Fonte: o Autor

A variável com a melhor correlação com o DAP foi o GE. Essa variável apresentou uma correlação significativa de $-0,86$ a $5 \%$ de probabilidade. Esse resultado indica que à medida que as árvores aumentam de diâmetro o GE diminui.

Silva et al. (2017) também obtiveram alta correlação negativa entre o DAP e o GE, sendo essa de $-0,81$, ressaltando que, com o aumento em diâmetro, o grau de esbeltez diminui e ocorre o aumento na estabilidade dos indivíduos.

O Diâmetro de Copa (DC) apresentou correlação de 0,80 com o DAP. Silva et al. (2017) afirmaram que o diâmetro de copa demonstra forte correlação com o DAP.

Já para a variável $\mathrm{H}$, a melhor correlação ocorreu com a variável CC, em que se observou uma correlação significativa de 0,37 a $1 \%$ de probabilidade. Esse resultado indica que a velocidade do crescimento apical acompanha linearmente o aumento da altura total nas árvores de Zanthoxylum rhoifolium.

Resultados semelhantes foram encontrados por Gardin (2017), estudando as variáveis morfométricas da espécie Curitiba prismatica (D. Legrand) Salywon \& Landrum, onde a melhor correlação com o DAP foi o GE, e a H com o CC.

O sinal negativo apresentado pelo coeficiente de correlação de Pearson entre o IS e o DAP indica que, de forma geral, o DC incrementa menos do que o DAP. Resultado semelhante foi encontrado por Tonini e Arco-Verde (2005), estudando Hymenaea courbaril L., onde a espécie também apresentou correlação negativa entre o IS com o DC, ou seja, quanto maior o IS menor será o DC.

Na Figura 3 são apresentados os modelos ajustados entre as variáveis Grau de Esbeltez (GE) e o Diâmetro a Altura do Peito (DAP) e entre o Diâmetro de Copa (DC), Diâmetro a Altura do Peito (DAP) respectivamente, que foram as que apresentaram as melhores correlações. $O$ grau de esbeltez apresentou correlação negativa com o DAP (Figura 3A). Com isso, à medida que aumenta o DAP, diminui o grau de esbeltez. 


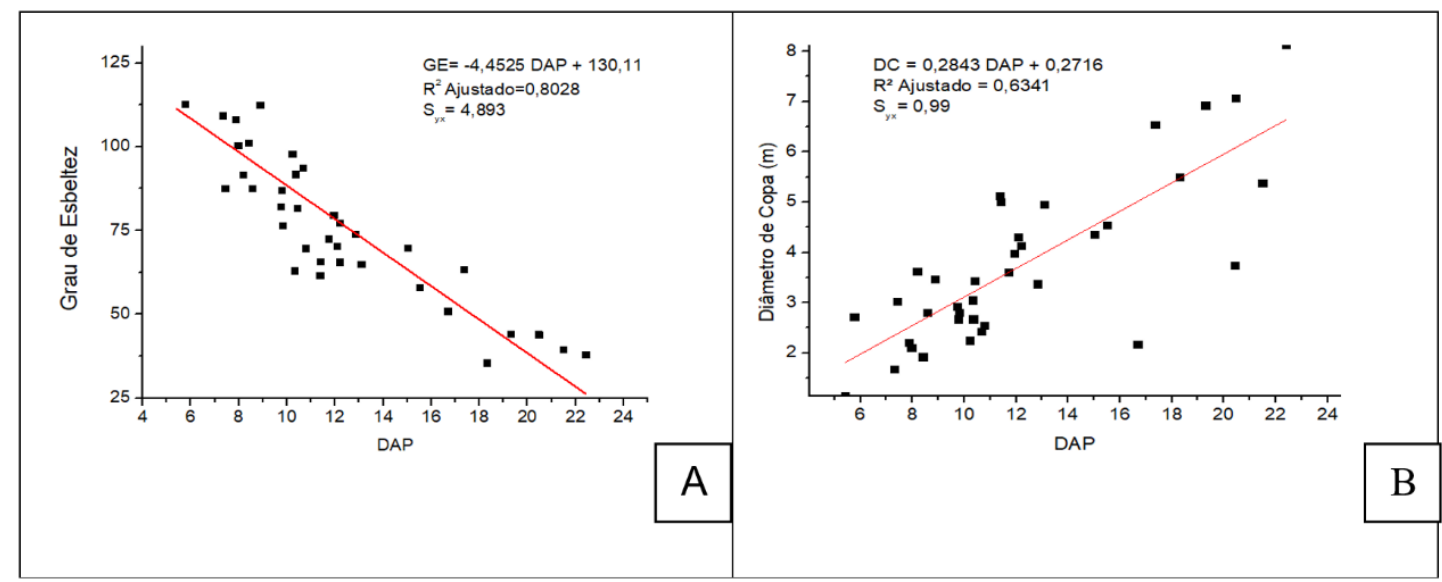

Figura 3. Modelo ajustado entre as variáveis Grau de Esbeltez e o Diâmetro a Altura do Peito (A) e entre diâmetro de copa e o Diâmetro a Altura do Peito (B) para Zanthoxylum rhoifolium em um fragmento de Floresta Ombrófila Mista Aluvial

Esse comportamento da diminuição do grau de esbeltez com o aumento do DAP também foi observado por Gardin (2017) para árvores de Curitiba prismatica. O diâmetro de copa de Z. rhoifolium relaciona-se significativamente com o DAP, tendendo a aumentar em razão do aumento do DAP (Figura 3B).

Curto (2015) estudando Araucaria angustifolia na Floresta Nacional de Açungui, Campo Largo, PR, relata que o desenvolvimento do diâmetro de copa em relação ao DAP segue padrão linear, ou seja, à medida que as árvores crescem em diâmetro, linearmente aumentase o diâmetro de copa, mas em algum determinado momento isto tende a se estabilizar.

\section{Análises Dendrocronológicas}

Com o programa COFECHA foi possível sincronizar, de maneira satisfatória, 10 árvores e 10 séries de um total de 37 árvores e 74 séries. As séries cronológicas excluídas apresentaram valores de intercorrelação abaixo do valor crítico, contribuíram para perda da correlação. A intercorrelação é o valor oriundo da comparação entre séries anuais de crescimento das árvores através do software COFECHA.

O valor de intercorrelação obtido na análise foi de 0,32 e o período considerado foi de 1973 a 2017, apresentando sensibilidade média de 0,435. A idade das árvores analisadas variou entre 9 a 44 anos (Tabela 3).

Tabela 3. Controle de qualidade das séries de anéis de crescimento pelo programa COFECHA para os indivíduos de Zanhoxylum rhoifolium em fragmento da Floresta Ombrófila Mista Aluvial.

\begin{tabular}{lcccccc}
\hline Espécie & $\begin{array}{c}\text { Número de } \\
\text { árvores/Séries } \\
\text { Antes } \\
\text { Depois }\end{array}$ & $\begin{array}{c}\text { Inter- } \\
\text { correlação } \\
\text { média }\end{array}$ & $\begin{array}{c}\text { Longitude } \\
\text { segmentos } \\
\text { (anos) }\end{array}$ & $\begin{array}{c}\mathbf{N}^{\circ} \text { de. anéis } \\
\text { observados }\end{array}$ & $\begin{array}{c}\text { Período } \\
\text { considerado }\end{array}$ & $\begin{array}{c}\text { Sensibilidade } \\
\text { Média }\end{array}$ \\
\hline $\begin{array}{l}\text { Zanhoxylum } \\
\text { rhoifolium }\end{array}$ & $\begin{array}{c}37(74) \\
10(10)\end{array}$ & 0,320 & $9-44$ & 341 & $1973-2017$ & 0,435 \\
\hline
\end{tabular}

Para espécies que crescem em locais que permitem a delineação dos períodos estivais e primaveris e os anéis de crescimento são nítidos, os valores de intercorrelação acima de 0,5 são considerados desejáveis, mas em regiões tropicais, em função das demarcações não serem visíveis, valores menores que 0,5 podem ser considerados valores aceitáveis (GrissinoMayer, 2001).

Guilhermeti (2015), estudando o crescimento de Gymnanthes klotzschiana Müll.Arg, na mesma área do presente estudo encontrou valores de intercorrelação de 0,52 para árvores de sub-bosque e 0,45 para as árvores do dossel. 
O valor da sensibilidade média indica a variabilidade inter-anual entre largura dos anéis de crescimento. Os valores acima de 0,30 indicam alta sensibilidade às mudanças ambientais (Grissino-Mayer, 2001). No presente trabalho, o valor de sensibilidade média foi 0,435, sendo assim, a espécie $Z$. rhoifolium apresenta alta sensibilidade às condições ambientais.

Guilhermeti (2015) encontrou valores de sensibilidade média superior a 0,32 para a espécie G. klotzschiana, ou seja, de alta sensibilidade. Para essa mesma espécie, Longhi-Santos (2013) obteve valor médio de 0,38.

As cronologias Master e Standard resultantes das análises nos softwares COFECHA e ARSTAN, respectivamente, para os indivíduos de Zanthoxylum rhoifolium estão apresentadas na Figura 4.

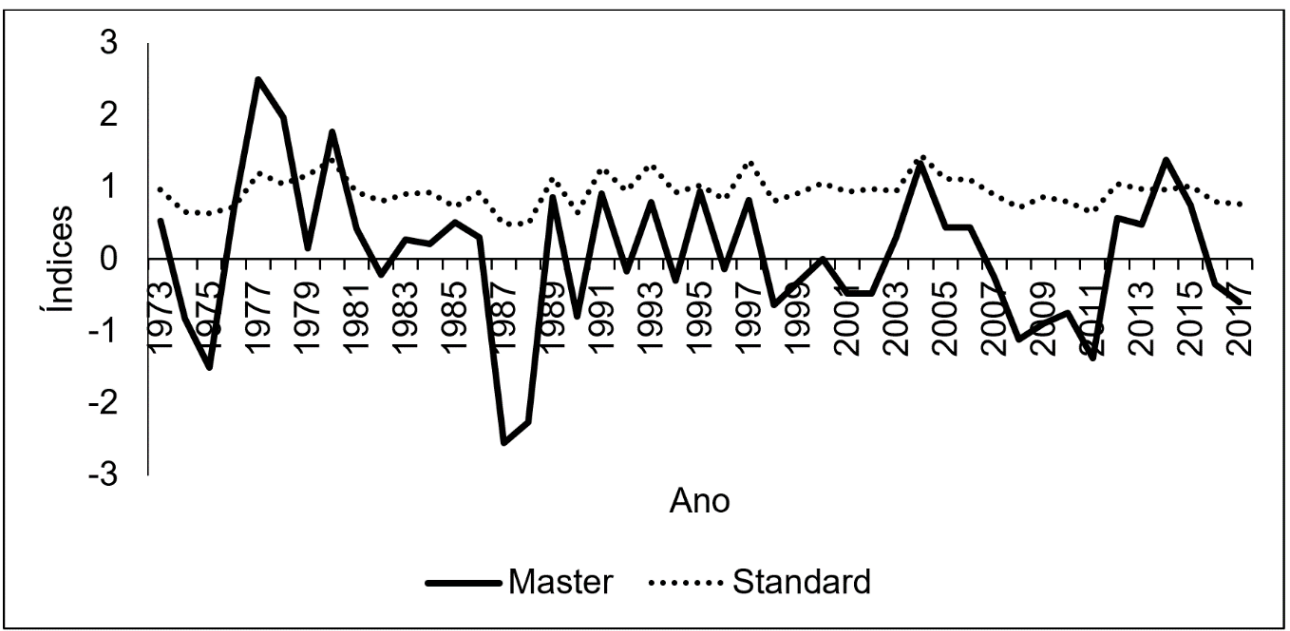

Figura 4. Índices de largura dos anéis de crescimento para Zanthoxylum rhoifolium, na série Master e Standard amostrados em fragmento da Floresta Ombrófila Mista Aluvial.

É possível observar na série Master que nos primeiros anos, de 1973 a 1987 ocorreram grandes oscilações no crescimento. Após este período, os índices tendem a ter um padrão mais homogêneo. Isto acontece, em consequência de haver um pequeno número de amostras sincronizadas nos primeiros anos. A partir do momento que a análise envolve um conjunto maior de amostras, os dados tendem a ter um padrão mais regular. Resultados semelhantes foram encontrados por Guilhermeti (2015), Kanieski et al. (2012) e Longhi-Santos (2013) em estudos dendrocronológicos de Gymnanthes klotzschiana.

A análise dos dados evidencia também que, a partir do ano de 1989, há uma elevação do índice de crescimento até o ano de 1998, depois ocorreu maior índice de incremento médio anual no período de 2004 a 2014.

\section{Correlação entre o crescimento de Zanthoxylum rhoifolium e as variáveis climáticas}

Comparando o incremento médio anual com as variáveis climáticas, verificou-se correlação significativa a nível de $1 \%$ de probabilidade com os dados de precipitação, como mostra a Tabela 4. As demais variáveis temperatura mínima, máxima, média e insolação não apresentaram correlações significativas.

Tabela 4. Correlação de Pearson entre o incremento médio anual de Zanthoxylum rhoifolium e as variáveis climáticas em um fragmento da Floresta Ombrófila Mista Aluvial.

\begin{tabular}{llllll}
\hline & $\begin{array}{l}\text { Temperatura } \\
\text { mínima }\end{array}$ & $\begin{array}{l}\text { Temperatura } \\
\text { máxima }\end{array}$ & $\begin{array}{l}\text { Temperatura } \\
\text { média }\end{array}$ & Precipitação & Insolação \\
\hline $\begin{array}{l}\text { Incremento } \\
\text { Médio Anual }\end{array}$ & 0,201 & $-0,189$ & 0,028 & $0,326^{* *}$ & $-0,199$ \\
\hline
\end{tabular}

Onde: * Correlação de Pearson significativa a um nível de 5\% de probabilidade; ** Significativa a um nível de $1 \%$ de probabilidade. 
A relação entre a média da precipitação anual e o incremento médio anual de $Z$. rhoifolium no período de 1977 a 2017. Verifica-se que no ano de 1983 ocorreu a maior precipitação média mensal (260 mm), e consequentemente maior saturação hídrica do solo, que pode ter dificultado o crescimento Z. rhoifolium (Figura 5). O maior crescimento da espécie Z. rhoifolium ocorreu no ano de 2004 , que pode ter sido beneficiada pela baixa precipitação neste ano (cerca de $150 \mathrm{~mm}$ ).

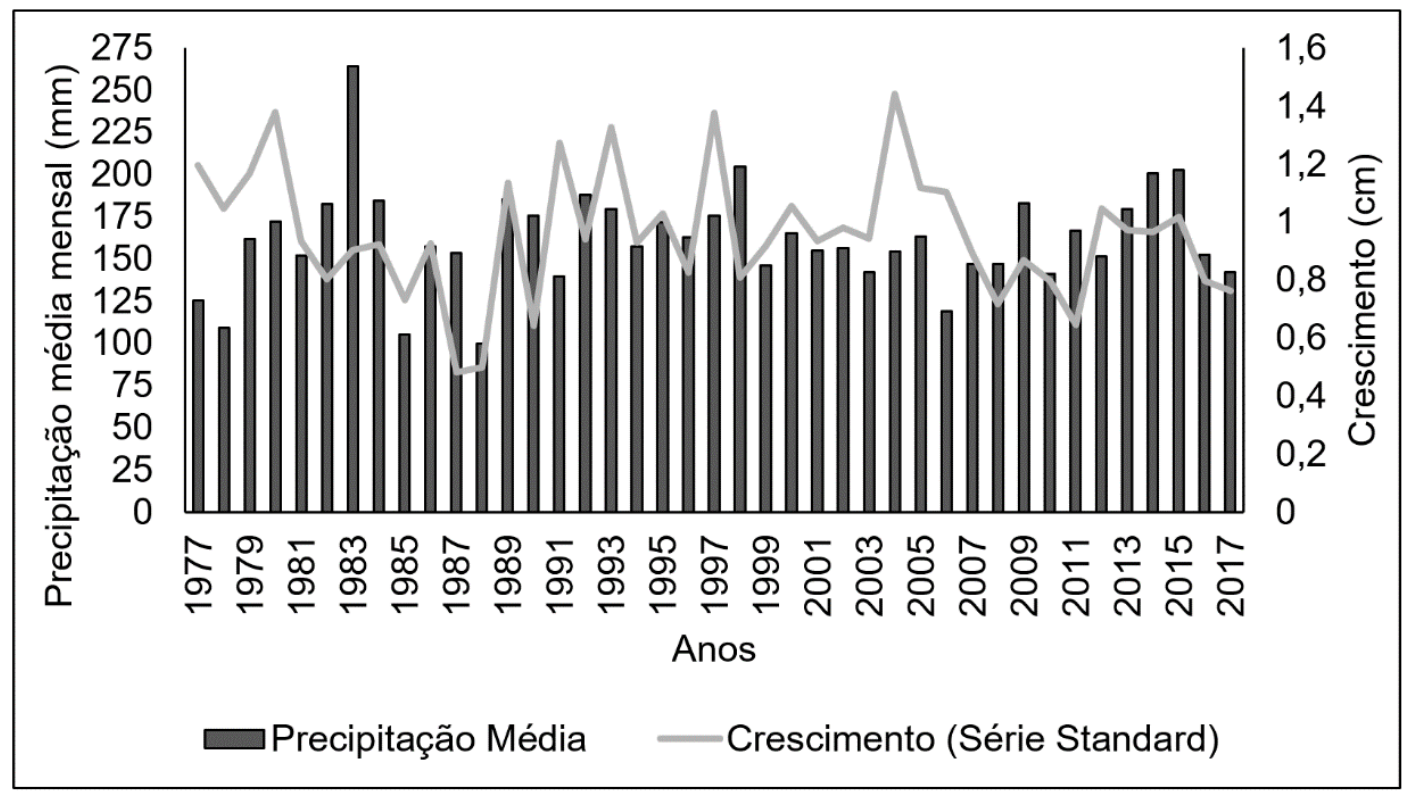

Figura 5- Relação entre a precipitação anual $(\mathrm{mm})$ e o índice de incremento médio annual da série Standard de Zanthoxylum rhoifolium em um fragmento de Floresta Ombrófila Mista Aluvial. Fonte: o Autor.

Nutto \& Watzlawick (2002) estudando a relação entre os fatores climáticos e o crescimento de Zanthoxylum rhoifolium não encontraram correlações significativas entre o crescimento e as variáveis temperatura e precipitação.

Zanon \& Finger (2010) avaliando a periodicidade do crescimento das árvores de Araucaria angustifolia, correlacionaram com as variáveis meteorológicas concluíram a temperatura e precipitação, estão diretamente correlacionadas com o incremento. 0 aumento da temperatura e da precipitação influem positivamente no incremento e a ocorrência de precipitação acompanhada de temperaturas baixas reduz o crescimento em diâmetro.

Outro fator importante que pode contribuir com a diminuição do crescimento de Zanthoxylum rhoifolium é o tipo de solo. Nas subunidades da área de estudo, os solos são hidromórficos com grande quantidade de argila, esta característica aliada ao fato de que o solo além de rochoso é muito raso, dificultam a drenagem do solo e favorecem o afloramento do lençol freático, mantendo o nível de água elevado e o solo saturado.

Cardoso et al. (2012) verificaram que o incremento radial das espécies variou segundo a saturação hídrica do solo. $\mathrm{O}$ incremento de Citharexylum myrianthum Cham. foi quatro vezes maior no Cambissolo, com maior drenagem, do que no Gleissolo, com saturação hídrica.

Vários estudos relatam a influência de variáveis meteorológicas como impulsionadores e/ou limitantes ao crescimento das espécies florestais. Alguns consideram a temperatura como fator que influencia o incremento diamétrico das árvores (Kanieski, 2013; LonghiSantos, 2013). Outros estudos consideram a precipitação e/ou disponibilidade de água no solo com maior influência sobre o incremento diamétrico (Cardoso et al., 2012; Nutto \& Watzlawick, 2002), em que a precipitação é extremamente importante para a retomada do crescimento, porém é limitante quando estiver em excesso no solo. 


\section{CONCLUSÕES}

- Os indivíduos de Zanthoxylum rhoifolium apresentaram altura total média de 8,43 m e DAP médio de $12,2 \mathrm{~cm}$.

- Zanthoxylum rhoifolium apresentou alta correlação do DAP com o grau de esbeltez e com o diâmetro de copa, sendo que a correlação entre o GE e DAP foi negativa, ou seja, à medida que a árvore aumenta em diâmetro, o GE diminui, indicando que a árvore é mais estável.

- O crescimento de Zanthoxylum rhoifolium apresentou correlação significativa com a precipitação média mensal.

\section{REFERÊNCIAS}

Burger, H. (1939). Baumkrone und Zuwachs in zwei hiebsreifen Fichtenbeständen. Mitteilungen der Schweizerischen Anstalt für das Forstliche Versuchswesen, 21, 147-176.

Cardoso, F. C. G., Marques, R., Botosso, P. C., \& Marques, M. C. M. (2012). Stem growth and phenology of two tropical trees in contrasting soil conditions. Plant and Soil, 354(1-2), 269-281. http://dx.doi.org/10.1007/s11104-011-1063-9.

Carvalho, P. E. R. (2006). Espécies arbóreas brasileiras (Vol. 1). Brasília: EMBRAPA Informação Tecnológica.

Condé, T. M., Lima, M. L. M., Lima Neto, E. M., \& Tonini, H. (2013). Morfometria de quatro espécies florestais em sistemas agroflorestais no município de Porto Velho, Rondônia. Revista. Agroambiente On-Line, 7(1), 18-27. http://dx.doi.org/10.18227/1982-8470ragro.v7i1.932.

Costa, E. A. (2011). Influência de variáveis dendrométricas e morfométricas no incremento diamétrico de Araucária Angustifolia (Bertol.) Kuntze, Lages, SC (Dissertação de mestrado). Universidade Federal de Santa Maria, Santa Maria.

Curto, R. A. (2015). Avaliação do crescimento e potencial de manejo em plantio superestocado de Araucaria angustifolia (Bert.) O. Ktze (Tese de doutorado). Universidade Federal do Paraná, Curitiba.

Durlo, M. A. (2001). Relações Morfométricas para Cabralea canjerana (Well.) Mart. Ciência Florestal, 11(1), 141-149. http://dx.doi.org/10.5902/19805098501.

Empresa Brasileira de Pesquisa Agropecuária - EMBRAPA. (2013). Sistema brasileiro de classificação de solos (3. ed. rev. ampl.). Brasilia.

Gardin, E. (2017). Aspectos ecofisiológicos e morfometria da Curitiba prismatica (D. Legrand) Salywon \& Landrum (Tese de doutorado). Universidade Estadual do Centro Oeste, Guarapuava.

Grissino-Mayer, H. D. (2001). Evaluating crossdating accuracy: a manual and tutorial for the computer program COFECHA. Tree-Ring Research, 57(2), 205-221.

Guilhermeti, P. G. C. (2015). Composição florística e crescimento de Branquilho em diferentes condições da Floresta Ombrófila Mista Aluvial (Dissertação de mestrado). Universidade Estadual do Centro Oeste do Paraná, Guarapuava.

Holmes, R. L. (1983). Computer-assisted control in tree-ring dating and mensurement. Tree-Ring Bulletin, $43,69-78$.

Homczinski, I. (2017). Distribuição espacial, dinâmica e biometria de Campomanesia xanthocarpa (Mart.) 0. Berg. em floresta ombrófila mista (Dissertação de mestrado). Universidade Estadual do CentroOeste, Irati.

Instituto Brasileiro de Geografia e Estatística - IBGE. (2012). Manual técnico da vegetação brasileira (275 p.). Rio de Janeiro.

Kanieski, M. R. (2013). Estudo dendroecológico de Sebastiania commersoniana (Baill.) L.B.Sm. \& R.J.Downs e de arbóreas invasoras em uma floresta ombrófila mista aluvial, sul do Brasil (Tese de doutorado). Universidade Federal do Paraná, Curitiba.

Kanieski, M. R., Santos, T. L., Graf Neto, J., Souza, T., Galvão, F., \& Roderjan, C. V. (2012). Influência da precipitação e da temperatura no incremento diamétrico de espécies florestais aluviais em Araucária-PR. Floresta e Ambiente, 19(1), 17-25. http://dx.doi.org/10.4322/floram.2012.003.

Lamprecht, H. (1990). Silvicultura nos trópicos: ecossistemas florestais e respectivas espécies arbóreas: possibilidades e métodos de aproveitamento sustentado (343 p.). Eschborn: Deustsche Gessells chaft fur technisch Zusammenarbeti (GTZ) GmbH. 
Lanzarim, K. (2016). Relações morfométricas e potencial de manejo de Maclura Tinctoria (L.) D. Don Ex. Steud em formações secundárias no noroeste do Rio Grande do Sul (Dissertação de mestrado). Universidade Federal de Santa Maria, Santa Maria.

Longhi-Santos, T. (2013). Dendroecologia de Sebastiania commersoniana (Baill.), LB Sm. \& Downs em um fragmento de floresta ombrófila mista aluvial, Paraná, Brasil (Dissertação de mestrado). Universidade Federal do Paraná, Curitiba.

Nutto, L., \& Watzlawick, L. F. (2002). Relações entre fatores climáticos e incremento em diâmetro de Zanthoxylum rhoifolia Lam. e Zanthoxylum hyemale St. Hil. na Região de Santa Maria, RS. Boletim de Pesquisa Florestal, 45, 41-55.

Orellana, E., \& Koehler, A. B. (2008). Relações morfométricas de Ocotea odorifera (Vell.) Rohwer. Revista Acadêmica Ciências Agrárias e Ambientais, 6(2), 229-237. http://dx.doi.org/10.7213/cienciaanimal.v6i2.10496.

Roman, M., Bressan, D. A., \& Durlo, M. A. (2009). Variáveis morfométricas e relações interdimensionais para Cordia trichotoma (Vell.) Arráb. Ex Steud. Ciência Florestal, 19(4), 473-480. http://dx.doi.org/10.5902/19805098901.

Santos, L. S., Lisi, C. S., Campelo, F., Vieira, J., \& Nabais, C. (2013). Aplicação das características das células vegetais como subsídio para a dendrocronologia: uma revisão. Belo Horizonte.

Silva, F. A., Riva, D., Fortes, F. O., \& Schorr, L. P. B. (2017). Caracterização de índices morfométricos para Araucaria angustifolia plantada na Região Norte do Rio Grande do Sul. Advances in Forestry Science, $4(3), 143-146$.

Silveira, B. D. (2014). Fitossociologia, crescimento e competição de três espécies nativas da floresta estacional semidecidual da região central do Rio Grande do Sul (Tese de doutorado). Universidade Federal do Paraná, Curitiba.

Tonini, H., \& Arco-Verde, M. F. (2005). Morfologia da copa para avaliar o espaço vital de quatro espécies nativas da Amazônia. Pesquisa Agropecuária Brasileira, 40(7), 633-638. http://dx.doi.org/10.1590/S0100-204X2005000700002.

Zanon, M. L. B., \& Finger, C. A. G. (2010). Relação de variáveis meteorológicas com o crescimento das ávores de Araucaria angustifolia (Bertol.) Kuntze em povoamentos implantados. Ciência Florestal, 20(3), 467-476. http://dx.doi.org/10.5902/198050982061.

Contribuição dos autores: APV: conceituação, curadoria de dados, análise formal, obtenção de financiamento, investigação, metodologia, administração do projeto, recursos, software, supervisão, validação, visualização, escrita - primeira redação, escrita - revisão e edição; ICB: conceituação, análise formal, investigação, metodologia, visualização, escrita - primeira redação, escrita - revisão e edição; TMZGS: conceituação, análise formal, investigação, metodologia, visualização, escrita - primeira redação, escrita - revisão e edição; LFW: conceituação, curadoria de dados, análise formal, obtenção de financiamento, investigação, metodologia, administração do projeto, supervisão, validação, visualização, escrita - primeira redação, escrita - revisão e edição; JAS: conceituação, investigação, metodologia, visualização, escrita - primeira redação, escrita - revisão e edição; BCP: investigação, visualização, escrita - primeira redação, escrita - revisão e edição. 